Research Article

\title{
Significance of Thermophoretic and Brownian Motion on MHD Nanofluids Flow towards a Circular Cylinder under the Inspiration of Multiple Slips: An Industrial Application
}

\author{
Aaqib Majeed $\mathbb{D},{ }^{1}$ Muhammad Zubair, ${ }^{2}$ Adnan Khan, ${ }^{2}$ Taseer Muhammad $\left(\mathbb{D},{ }^{3}\right.$ \\ and M.S. Alqarni ${ }^{3}$ \\ ${ }^{1}$ Department of Mathematics, Riphah International University, Faisalabad Campus, Faisalabad 38000, Pakistan \\ ${ }^{2}$ Department of Mathematics, University of Peshawar, Peshawar 25120, Khyber Pakhtunkhwa, Pakistan \\ ${ }^{3}$ Department of Mathematics, College of Sciences, King Khalid University, Abha 61413, Saudi Arabia \\ Correspondence should be addressed to Taseer Muhammad; taseer_qau@yahoo.com
}

Received 7 June 2021; Revised 28 August 2021; Accepted 7 September 2021; Published 13 October 2021

Academic Editor: Constantin Fetecau

Copyright (C) 2021 Aaqib Majeed et al. This is an open access article distributed under the Creative Commons Attribution License, which permits unrestricted use, distribution, and reproduction in any medium, provided the original work is properly cited.

In this article, MHD flow of silver/water nanofluid past a stretched cylinder under the impact of thermal radiation with chemical reaction and slip condition is studied. The impact of Soret and Dufour effect is also analyzed during this flow. The uniqueness of the given problem is enlarged with the insertion of variable magnetic field, free stream velocity, thermal slip condition, and nonlinear thermal radiation. The PDEs are converted to ODEs by using suitable similarity transformation. The nonlinear system of ODEs is solved by applying convergent homotopy analysis method (HAM). The velocity, temperature, and concentration profiles for the free stream and at the plate are discussed through graphs and numerical tables. It is found that velocity field reduces, while the temperature profile rises for the increasing values of magnetic parameter. It is examined that effects of curvature on frication factor are increasing. Furthermore, temperature profile increases for greater Brownian motion and thermophoresis parameters. Transfer of heat enhances decreasing the radius of the cylinder also with heat generation parameter. The skin friction can be reduced by enhancing free stream and wall stretching velocities ratio. Velocity profile of the flow can be controlled by enhancing velocity slip and magnetic field.

\section{Introduction}

It is acknowledged that the progress in the human culture mostly depends on the sources of energy. Therefore, energy is utilized by the researchers in the universe for various functions so that the human being can take benefit from it. The sun is a source of energy which is used by the specialists and researchers in different technologies for the purpose of thermal conductivity and convective heat transfer. When the solar energy reaches the Earth, it is approximately $\left(4 \times 10^{15} \mathrm{MW}\right)$. Solar energy is 2000 times more powerful than the world's total energy use. Choi [1] was the first to introduce the term nanofluid. Nanofluids are made up of nanoparticles and base fluid flows. Choi came to the conclusion that a relatively small amount of nanoparticles can have a huge impact on heat generation processes. Choi discovered that, by utilizing silver nanoparticles, the thermal conductivity and convective heat of liquid will be double. Nanoparticles can be round, rod-like, or tabular in shape. The impact of Soret and Dufour effect on MHD for the study of convective radiation and mass transfer was studied by Pal et al. [2]. MHD is abbreviated as the magnetohydrodynamics, which deals with the study of magnetic properties and the behavior of electrically conductive fluid. Examples of such magnetofluids are plasma, liquid metals, salt water, and electrolytes. The basic idea behind the MHD is that magnetic fields can induce current in a movable conductive fluid, which in turn polarizes the fluid and reciprocally changes the magnetic field itself. Hussain et al. [3] investigated the magnetic movement of Jeffrey nanoparticles caused by 
increasing the power overstretched layer in the presence of heat resistance. Impact of MHD on the porous medium was studied by Bhattacharyya and Layek [4]. Rashidi et al. [5] investigated the behavior of magnetic flow of nanoparticles sizes with the generation of thermal heat due to the rotatory motion of the disk. Mabood and Mastroberardino [6] also examined the behavior of nanoparticles and heat transport of magnetic field. Hayat et al. [7] studied the impact of magnetic field to obtain the equilibrium viscosity in the nanofluid and talked about the solar heating system and its heat generation. Shafiq et al. [8] studied the influence of nonlinear thermal radiation on magnetohydrodynamic (MHD) Darcy-Forchheimer Casson-water nanofluid flow on a circular disk. Rasool and Shafiq [9] worked on the MHD, heat sink/source, and convective boundary conditions in artificially receptive radiative Powell-Eyring nanofluid stream through Darcy channel utilizing a nonlinearly extending sheet/surface. A double substance response term is considered in the model. Nonlinear radiation is accounted for inside the stream. The model includes impact of warmth sink/source. Alotaibi et al. [10] presented the impact of heat generation and suction on magnetohydrodynamic (MHD) boundary layer flow of Casson nanofluid on a nonstretching surface in two dimensions. Abd Elazem and Nader [11] demonstrated that, because of its critical applications in physical science, chemistry, and designing, some interest has been given lately to investigating the boundary layer stream of magnetohydrodynamic nanofluids. The mathematical outcomes were discussed for temperature profile, concentration profile, decreased Nusselt number, and reduced Sherwood number.

One of the most important elements affecting convective heat transfer in nanofluid is its increased thermal conductivity. As a result, it is critical to investigate the notion of thermal conductivity in order to develop a good heat exchange system that allows for easy heat transfer. Thermal conductivity of silver-based nanofluid is the highest as compared to other nanofluids. The results illustrate that cylindrical nanoparticles deliver the highest thermal conductivity compared to spherical particles; also it is noticed that all particles enhance heat transfer. But if the size of nanoparticles is too small, then agglomeration takes places and again thermal conductivity decreases. Muhammad et al. [12] discussed mixed convective flow and heat with mass transfer analysis towards stretchable surface. Lio [13] considered the flow towards a permeable surface with variable thermal conductivity. Water is the most suitable selection for the cooling applications in the processes to transfer heat as it has very high heat capacity and thermal conductivity. Nanofluid has some specific property which made it suitable in many industrial and heat transfer applications [14]. The presence of magnetic field, thermophoresis, and Brownian motion is considered to contemplate the model of flow. Waqas et al. [15] mentioned the method of heat conversation in industrial usages based on the convective heat transfer which are enriched by using porous medium and magnetic field. Khan et al. [16] worked on the effect of Maxwell fluid flow on vertically upward or downward rotating disk and considered the unsteady motion in the existence of magnetic flux. Ramesh et al. [17] discussed the application of hybrid nanofluid along with the heat transfer effect for the stretched cylinders. Ramzan et al. [18] studied the heat generation or absorption and found the important impact of heat exchange rate. Sarwe and Kulkarni [19] explored the steady state of heat exchange and thermal examination of hyperbolic annular fin with the heat dependent thermal conductivity. Hosseinzadeh et al. [20] examined the chemically responsive transmitting stream by utilizing a two-dimensional Darcy-Forchheimer model with the convectively heated plate. The nonlinear thermal radiation is defined by Joule hating effect. Kumar et al. [21] studied the effect of thermophoretic molecule deposition and attractive dipole in the progression of Maxwell fluid on an extending sheet. Kumar et al. [22] explored to talk about the progression of a ferromagnetic viscous fluid with thermophoretic molecule deposition over an extending chamber on account of uniform heat source/sink. Venthan et al. [23] studied the progress of Bingham nanofluids at the entrance segment working as non-Newtonian nanofluids in cylindrical shaped concentric rollers have been examined. This examination could be beneficial for substance handling productions and intensified thermal preparing. Rehman et al. [24] studied the mathematical methodology to investigate the examination of associated convection and heat radiation on atomic hypothesis of fluid started nanofluid over an extendable surface.

Non-Newtonian fluids are those fluids which do not obey Newtonian law of viscosity and they vary as shear stress is proportional to the velocity gradient such that they vary to the power $N$, where its value will not be equal to one. They are used in ink, paper pulp, wax, tomato sauce or ketchup, corn starch in water solution golden syrup, or honey, and the most obvious case is honey, which does not flow like water, coffee of solution in water, motor oil, brake fluid or clutch fluid (hydraulic fluids), for lubrication of spray. NonNewtonian fluids are mentioned in three different ways: first is the rate type, the second one is the differential way, and the third one is a lot as integral way. In Maxwell description, the rate type has very limited scope due to its characteristic of relaxation time. While Jeffery analysis of rate type is more valid, it deals with the dual nature property, that is, relaxation and retardation of time. So, this model has the viscoelastic property which has more benefit in polymers industries $[25,26]$. When the relation between heat transfer and local temperature is directly proportional, we call this statement Newtonian heating; it may also be referred to as the heat flowing between a surface and fluid inside it or outside the surface, which will occur as a result of colliding objects. Merkin [27] discussed the heat transfer phenomena in a list of four: firstly Newtonian heating, secondly the boundary condition of heat transfer rate, thirdly the heat flux for the constant surface, and the last one is for surface temperature. There are a lot of researcher works on the study of Newtonian heating [25, 28-31].

Acharya et al. [32] explored the impact of high-order chemical reaction and second-order slip on the bioconvective nanofluid involving of gyrotactic microorganism between two pressed parallel plates. For more relevant 
studies, see [33, 34]. Rasool et al. [35] studied the behavior of chemical reaction, thermal radiations, and Soret-Dufour influence on a steady incompressible Darcy-Forchheimer flow of nanofluids. Hosseinzadeh et al. [20] examined the chemically responsive transmitting stream by utilizing a two-dimensional Darcy-Forchheimer model with the convectively heated plate. The nonlinear thermal radiation is defined by Joule hating effect.

By keeping the above literature review in mind, an abundance of work is present on the nanofluid flow past a stretched cylinder but a little bit where the free stream velocity is function of spatial variable. Now here we analyzed such kind of flow under the impact of MHD, thermal radiation, and heat source. The chemical reaction is also kept in mind for the best examination in heat and mass transmission near the stagnation point. The modeled coupled set of ODEs are solved by semianalytical HAM. The graphs of the obtained results are drawn in computer-based package Mathematica and discussed comprehensively.

\section{Mathematical Formulation}

Here we considered 2D flow of Silver-Water nanofluid on a stretched cylinder under the impact of $\mathrm{MHD}$, as can be seen in Figure 1. We assumed that the flow is steady and incompressible. In current research, the effects of chemical reaction and thermal radiation were also analyzed. In the mathematical modeling of the problem, the velocity and thermal slip boundary conditions are also taken into account. The constant magnetic field having strength $B_{0}$ is applied in the direction of cylinder radius. Here we assumed very low Reynolds number and therefore induced magnetic and electric fields are ignored. The governing equations of the flow are as follows [36-38]:

$$
\begin{aligned}
& \frac{\partial(r u)}{\partial x}+\frac{\partial(r v)}{\partial r}=0, \\
& u \frac{\partial u}{\partial x}+v \frac{\partial u}{\partial r}=U_{e} \frac{d U_{e}}{d x}+v_{n f}\left(\frac{\partial^{2} u}{\partial r^{2}}+\frac{1}{r} \frac{\partial u}{\partial r}\right)-\frac{\sigma_{n f}}{\rho_{n f}} B_{0}^{2} u, \\
& \left.\begin{array}{c}
\frac{\partial T}{\partial x}+v \frac{\partial T}{\partial r}= \\
\alpha_{n f}\left(\frac{\partial^{2} T}{\partial r^{2}}+\frac{1}{r} \frac{\partial T}{\partial r}\right)+\frac{\mu_{n f}}{\left(\rho c_{p}\right)_{n f}}\left(\frac{\partial u}{\partial r}\right)^{2}+ \\
\tau\left[D_{B} \frac{\partial T}{\partial r} \frac{\partial C}{\partial r}+\frac{D_{T}}{T_{\infty}}\left(\frac{\partial T}{\partial r}\right)^{2}\right]+\frac{Q_{0}}{\left(\rho c_{p}\right)_{n f}}\left(T-T_{\infty}\right)-\frac{1}{\left(\rho c_{p}\right)_{n f}} \frac{\partial q_{r}}{\partial r}
\end{array}\right\} \\
& u \frac{\partial C}{\partial x}+v \frac{\partial C}{\partial r}=D_{B}\left(\frac{\partial^{2} C}{\partial r^{2}}+\frac{1}{r} \frac{\partial C}{\partial r}\right)+\frac{D_{T}}{T_{\infty}}\left(\frac{\partial T}{\partial r^{2}}+\frac{1}{r} \frac{\partial T}{\partial r}\right)-k_{C}\left(C-C_{\infty}\right) .
\end{aligned}
$$

The boundary conditions of the problem are like

$$
\begin{aligned}
& \text { at } r \longrightarrow R, u(x, r)=U_{w}(x)+L \frac{\partial u}{\partial r}, \\
& v(x, r)=0, \\
& T(x, r)=T_{w}(x)+K_{1} \frac{\partial T}{\partial r}, \\
& C(x, r)=C_{w}(x)+K_{2} \frac{\partial C}{\partial r},
\end{aligned}
$$

$$
\begin{aligned}
\text { at } r & \longrightarrow \infty, u(x, r) \longrightarrow U_{e}, \\
T(x, r) & \longrightarrow T_{\infty}, \\
C(x, r) & \longrightarrow C_{\infty} .
\end{aligned}
$$

Here

$$
\left.\begin{array}{l}
U_{w}(x)=\frac{U_{0} x}{l}, T_{w}(x)=T_{0}+\frac{a x}{l}, C_{w}(x)=C_{0}+\frac{b x}{l}, \text { at } r \longrightarrow R, \\
U_{e}=\frac{U_{\infty} x}{l}, T_{\infty}=T_{0}+\frac{d x}{l}, C_{\infty}=C_{0}+\frac{e x}{l}, \text { at } r \longrightarrow \infty .
\end{array}\right\}
$$




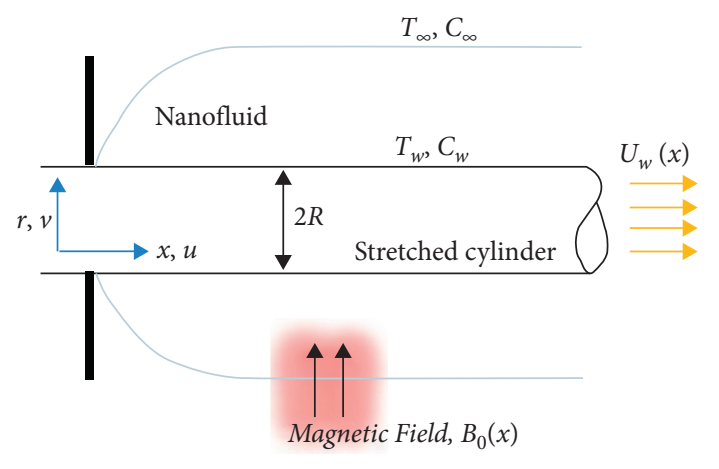

Figure 1: Geometrical representation of the flow.

The velocity components in the form of stream functions are defined as

$$
\begin{aligned}
& u=-\frac{1}{r} \frac{\partial \psi}{\partial x}, \\
& v=+\frac{1}{r} \frac{\partial \psi}{\partial r}, \quad \text { where } \psi=\sqrt{\frac{U_{0} v x^{2}}{l}} R f(\eta) .
\end{aligned}
$$

\section{Similarity Transformations}

To transform the problem from PDEs to ODEs, we choose the similarity transformations like [36]

$$
\begin{aligned}
u & =\frac{U_{0}}{l} x f^{\prime}(\eta), \\
v & =-\sqrt{\frac{U_{0} v}{l}} \frac{a}{r} f(\eta), \\
T(\eta) & =\frac{T-T_{\infty}}{T_{w}-T_{0}} \\
\phi(\eta) & =\frac{C-C_{\infty}}{C_{w}-C_{0}}, \\
\text { Here } \eta & =\left(\frac{r^{2}-R^{2}}{2 R}\right) \sqrt{\frac{U_{0}}{v l}}
\end{aligned}
$$

Here $u$ and $v$ are the velocity components along the $x$ axis and $r$-axis, respectively. The momentum profile of the flow is controlled by the physical parameters symbolized as $U_{e}=U_{\infty} x / l, v_{n f}, \alpha_{n f}, \rho_{n f}$, and $B_{0}$ which are free stream velocity, kinematic viscosity of nanofluid, thermal diffusivity of the nanofluid, and density and strength of magnetic field, respectively. In the energy equation, the controlling parameters are $T, \mu_{n f}, \tau, D_{B}, D_{T}, q_{r}$ denoting the temperature profile, dynamic viscosity of nanofluid, shear stress, Brownian diffusion coefficient, thermophoresis diffusion parameter, and radiation of heat flux, respectively. In the concentration equation, $C, K_{c}, C_{\infty}$, and $T_{\infty}$ express the concentration profile, coefficient of chemical reaction, ambient concentration, and ambient temperature, respectively. The thermophysical characteristics of the nanoparticles and base fluid are declared in Table 1, respectively.

Using the similarity transformation given in equations (8) and (9) into equations (1)-(4), the continuity equation (1) is identically satisfied, while equations (2)-(4) are transformed from PDEs to ODEs in the following forms:

$$
\begin{aligned}
& \left(1-\varphi+\varphi \frac{\rho_{s}}{\rho_{f}}\right)(1-\varphi)^{2.5} A^{2}+(1+2 \gamma \eta) f^{\prime \prime \prime}+2 \gamma f^{\prime \prime}+ \\
& \left.\begin{array}{l}
\left(1-\varphi+\varphi \frac{\rho_{s}}{\rho_{f}}\right)(1-\varphi)^{2.5}\left(f f^{\prime \prime}-f^{\prime 2}\right)-(1-\varphi)^{2.5} M f^{\prime}=0,
\end{array}\right\} \\
& (1+2 \gamma \eta)\left[\frac{k_{n f}}{k_{f}}+(1+(N r-1) \theta)^{3}\right] \theta^{\prime \prime}+\left[\frac{1}{2 K^{*}} \frac{k_{n f}}{k_{f}}+(1+(N r-1) \theta)^{3}\right] \gamma \theta^{\prime} \\
& +\frac{\operatorname{Pr}}{4 K^{*}}\left[1-\varphi+\varphi \frac{\left(\rho c_{p}\right)_{s}}{\left(\rho c_{p}\right)_{f}}\right]\left[f \theta^{\prime}-f^{\prime} \theta+D c \theta+\frac{E c}{(1-\varphi)^{2.5}} f^{\prime \prime 2}\right]+2(1+2 \gamma \eta)(N r-1)(1+(N r-1) \theta)^{2} \theta^{\prime 2} \\
& +\operatorname{Pr}(1+2 \gamma \eta)\left(N b \theta^{\prime} \phi^{\prime}+N t \theta^{\prime 2}\right)=0, \\
& \quad(1+2 \gamma \eta) \phi^{\prime \prime}+2 \gamma \phi^{\prime}+S c f \phi^{\prime}+\frac{N t}{N b}\left((1+2 \gamma \eta) \theta^{\prime \prime}+2 \gamma \theta^{\prime}\right)-\operatorname{Pr} L e\left(f^{\prime} \phi+\epsilon_{2} f^{\prime}+\epsilon_{3} \phi^{\prime}\right)=0 .
\end{aligned}
$$

Apply the transformations given in equations (8) and (9) on boundary conditions given in equations (5) and (6). After the transformation and simplification, the boundary conditions take the following form: 
TABLE 1: Thermophysical properties of the nanoparticles with base fluid [39].

\begin{tabular}{lcc}
\hline Thermophysical characteristics & Water & Ag \\
\hline$C_{p}\left(\mathrm{Jkg}^{-1} \mathrm{~K}^{-1}\right)$ & 4179 & 235.0 \\
$\rho\left(\mathrm{kgm}^{-3}\right)$ & 997.1 & 10500.0 \\
$\kappa\left(\mathrm{WmK}^{-1}\right)$ & 0.61300 & 429.0 \\
\hline
\end{tabular}

$$
\left.\begin{array}{l}
r \longrightarrow R: f(0)=0, f^{\prime}(0)=1+S_{1} f^{\prime \prime}(0), \theta(0)=1-\epsilon_{1}+S_{2} \theta^{\prime}(0), \phi(0)=1-\epsilon_{2}+S_{3} \phi^{\prime}(0), \\
r \longrightarrow \infty: f^{\prime}(\infty)=A, \theta(\infty)=0, \phi(\infty)=0,
\end{array}\right\}
$$

where

$$
\begin{aligned}
& \gamma=\left(\frac{v l}{U_{0} R^{2}}\right)^{\frac{1}{2}}, \\
& A=\frac{U_{\infty}}{U_{0}}, \\
& S c=\frac{v}{D_{B}}, \\
& S_{1}=L \sqrt{\frac{U_{0}}{v l}}, \\
& S_{2}=K_{1} \sqrt{\frac{U_{0}}{v l}}, \\
& S_{3}=K_{2} \sqrt{\frac{U_{0}}{v l}}, \\
& \operatorname{Pr}=\frac{\mu c_{p}}{k}, \\
& N t=\tau D_{T} \frac{\left(T_{w}-T_{\infty}\right)}{v T_{\infty}}, \\
& N b=\tau D_{B} \frac{C_{w}-C_{\infty}}{v}, \\
& \epsilon_{1}=\frac{d}{a} \\
& \epsilon_{2}=\frac{e}{b}, \\
& \epsilon_{3}=\frac{k_{C} l}{U_{0}} \\
& M=\frac{\sigma B_{0}^{2}}{U_{0} \rho_{f}}, \\
& K^{*}=\frac{16 \sigma^{*}}{3 k^{*} k} T_{\infty}^{3}, \\
& N r=\frac{T_{w}}{T_{\infty}}, \\
& E c=\frac{U_{w}^{2}}{c_{p} T_{\infty}}, \\
& D c=\frac{Q_{0}}{U_{0}\left(\rho c_{p}\right)_{f}}, \\
& L e=\frac{k / \rho c_{p}}{D_{B}} .
\end{aligned}
$$

The physical parameters in the above nondimensional equations are symbolized as $\gamma, A, S c, S_{i}(i=1,2,3), L e, P r$, $N t, N b, M, K^{*}, N r, E c$, and $D c$ these parameters are curvature parameter, ratio of the velocities, Schmidt number, slip parameter of the velocity, the Lewis number, Prandtl number, the thermophoretic parameter, Brownian motion parameter, magnetic parameter, mean absorption coefficient, temperature ratio parameter, Eckert number, and heat generation parameter, respectively. The applied numerical quantities having the great importance at industrial level are the local skin fraction $C_{f}$, local Nusselt number $N u_{x}$, and the local Sherwood numbers $S h_{x}$. These physical quantities are defined mathematically as

$$
\begin{aligned}
C_{f} & =\frac{2 \tau_{w}}{\rho U_{w}^{2}}, \\
N u_{x} & =\frac{x q_{w}}{\kappa\left(T_{w}-T_{\infty}\right)}, \\
S h_{x} & =\frac{x q_{m}}{D\left(C_{w}-C_{\infty}\right)},
\end{aligned}
$$

where $\tau_{w}, q_{w}$, and $q_{m}$ represent shear stress, heat flux, and mass flux, respectively, and are given by

$$
\begin{aligned}
& \tau_{w}=\left.\mu_{n f} \frac{\partial u}{\partial r}\right|_{r=R}, \\
& q_{w}=-\left.\kappa k_{n f}\left(1+\frac{4 \sigma^{*} T_{\infty}^{3}}{3 k^{*}}\right) \frac{\partial T}{\partial r}\right|_{r=R}, \\
& q_{m}=-\left.D \frac{\partial C}{\partial r}\right|_{r=R} .
\end{aligned}
$$

Using the above similarity variable given in equation (9) and after the simplification, we have

$$
\begin{aligned}
\frac{1}{2} C_{f} \operatorname{Re}_{x}^{1 / 2} & =\frac{1}{(1-\varphi)^{2.5}} f^{\prime \prime}(0), \\
N u_{x} \operatorname{Re}_{x}^{-1 / 2} & =-\frac{k_{n f}}{k_{f}}\left(1+K^{*}\right) \theta^{\prime}(0), \\
S h_{x} \operatorname{Re}_{x}^{-1 / 2} & =-\phi^{\prime}(0) .
\end{aligned}
$$

Here, $\operatorname{Re}_{x}=U_{w} x / v$ represents the Reynolds number. 


\section{Solution Procedure}

In 1992, Liao [44] firstly worked on the homotopy analysis method (HAM). By this method, we solve a system of coupled highly nonlinear differential equations. For performing this technique, we assumed the initial guesses $\left(f_{0}, \theta_{0}, \phi_{0}\right)$ and the linear operators $\left(\left\langle_{f}\left\langle_{\theta}\left\langle_{\phi}\right)\right.\right.\right.$ such as

$$
\begin{aligned}
& f_{0}(\eta)=A \eta+\frac{1-A}{1+S_{1}}\left(1-e^{-\eta}\right), \\
& \theta_{0}(\eta)=\frac{1}{1+S_{2}} e^{-\eta}, \\
& \phi_{0}(\eta)=\frac{1}{1+S_{3}} e^{-\eta} .
\end{aligned}
$$

The linear operators for the solution are like

$$
\begin{aligned}
& \left\langle f(f)=\frac{d^{3} f}{d \eta^{3}}-\frac{d f}{d \eta},\right. \\
& \left\langle\theta(\theta)=\frac{d^{2} \theta}{d \eta^{2}}-\theta,\right. \\
& \left\langle\phi(\phi)=\frac{d^{2} \phi}{d \eta^{2}}-\phi,\right.
\end{aligned}
$$

and further

$$
\begin{aligned}
& N_{\widehat{f}}[\hat{f}(\eta ; \zeta), \widehat{\phi}(\eta ; \zeta)]=\left(1-\varphi+\varphi \frac{\rho_{s}}{\rho_{f}}\right)(1-\varphi)^{2.5} A^{2}+(1+2 \gamma \eta) \hat{f}_{\eta \eta \eta}+2 \gamma \hat{f}_{\eta \eta}+ \\
& N_{\widehat{\theta}}[\widehat{f}(\eta ; \zeta), \widehat{\theta}(\eta ; \zeta), \widehat{\phi}(\eta ; \zeta)]=(1+2 \gamma \eta)\left[\frac{k_{n f}}{k_{f}}+(1+(N r-1) \hat{\theta})^{3}\right] \hat{\theta}_{\eta \eta} \\
& +\left[\frac{1}{2 K^{*}} \frac{k_{n f}}{k_{f}}+(1+(N r-1) \bar{\theta})^{3}\right] \gamma \bar{\theta}_{\eta}+\frac{\operatorname{Pr}}{4 K^{*}}\left[1-\widehat{\phi}+\widehat{\phi} \frac{\left(\rho_{c p}\right)_{s}}{\left(\rho_{c p}\right)_{f}}\right] \\
& \cdot\left[\hat{f} \hat{\theta}_{\eta}-\hat{f}_{\eta}+D c \hat{\theta}+\frac{E c}{(1-\varphi)^{2.5}} \hat{f}_{\eta \eta}^{2}\right] \\
& +2(1+2 \gamma \eta)(N r-1)\left(1+(N r-1) \widehat{\theta}^{2} \widehat{\theta}_{\eta}{ }^{2}+\operatorname{Pr}(1+2 \gamma \eta)\left(N b \widehat{\theta}_{\eta} \widehat{\phi}_{\eta}+N t \widehat{\theta}_{\eta}^{2}\right)\right. \\
& \left.N_{\widehat{\phi}}[\hat{\phi}(\eta ; \zeta), \hat{f}(\eta ; \zeta), \hat{\theta}(\eta ; \zeta)]=(1+2 \gamma \eta) \hat{\phi}_{\eta \eta}+2 \gamma \widehat{\phi}_{\eta}+\frac{N t}{N b}(1+2 \gamma \eta) \hat{\theta}_{\eta \eta}+2 \gamma \widehat{\theta}_{\eta}\right)+\operatorname{Pr} L e \hat{\phi} \\
& \left(1-\varphi+\varphi \frac{\rho_{s}}{\rho_{f}}\right)(1-\varphi)^{2.5}\left(\hat{f}_{\bar{f}}-\hat{f}^{\prime 2}\right)-(1-\varphi)^{2.5} M \hat{f}_{\eta} .
\end{aligned}
$$

For equations (7)-(9), the $0^{\text {th }}$ order solution is written as

$$
\begin{aligned}
& (1-\zeta) L_{\widehat{f}}\left[\hat{f}(\eta ; \zeta)-\hat{f}_{0}(\eta)\right]=p h_{\widehat{f}} N_{\widehat{f}}[\widehat{f}(\eta ; \zeta), \widehat{\theta}(\eta ; \zeta),(\eta ; \zeta)], \\
& (1-\zeta) L_{\hat{\theta}}\left[\hat{\theta}(\eta ; \zeta)-\hat{\theta}_{0}(\eta)\right]=p h_{\hat{\theta}} N_{\hat{\theta}}[\hat{f}(\eta ; \zeta), \hat{\theta}(\eta ; \zeta), \hat{\phi}(\eta ; \zeta)], \\
& (1-\zeta) L_{\hat{\phi}}\left[\hat{\phi}(\eta ; \zeta)-\widehat{\phi}_{0}(\eta)\right]=p h_{\hat{\phi}} N_{\hat{\phi}}[\hat{f}(\eta ; \zeta), \hat{\theta}(\eta ; \zeta), \widehat{\phi}(\eta ; \zeta)] .
\end{aligned}
$$


For subjected boundary conditions, we have

$$
\begin{aligned}
& \left.\hat{f}(\eta ; \zeta)\right|_{\eta=0}=0, \\
& \left.\frac{\partial \hat{f}(\eta ; \zeta)}{\partial \eta}\right|_{\eta=0}=1, \\
& \left.\hat{\theta}(\eta ; \zeta)\right|_{\eta=0}=1, \\
& \left.\frac{\partial \hat{\theta}(\eta ; \zeta)}{\partial \eta}\right|_{\eta=0}=1, \\
& \left.\frac{\partial \hat{\theta}(\eta ; \zeta)}{\partial \eta}\right|_{\eta=\infty}=A, \\
& \left.\hat{\theta}(\eta ; \zeta)\right|_{\eta=\infty}=0 . \\
& \left.\hat{\theta}(\eta)\right|_{\eta=\infty}=0,
\end{aligned}
$$

and Taylor's expansion for $\hat{f}(\eta ; \zeta), \hat{\theta}(\eta ; \zeta)$, and $\hat{\phi}(\eta ; \zeta)$ about $\zeta=0$ is

$$
\left.\begin{array}{l}
\hat{f}(\eta ; \zeta)=\hat{f}_{0}(\eta)+\sum_{n=1}^{\infty} \hat{f}_{n}(\eta) \zeta^{n}, \\
\hat{\theta}(\eta ; \zeta)=\hat{\theta}_{0}(\eta)+\sum_{n=1}^{\infty} \hat{\theta}_{n}(\eta) \zeta^{n}, \\
\hat{\phi}(\eta ; \zeta)=\hat{\phi}_{0}(\eta)+\sum_{n=1}^{\infty} \hat{\phi}_{n}(\eta) \zeta^{n},
\end{array}\right\}
$$

where

$$
\begin{aligned}
& \hat{f}_{n}(\eta)=\frac{1}{n !} \frac{\partial \hat{f}(\eta ; \zeta)}{\partial \eta} \\
& \hat{\theta}_{n}(\eta)=\frac{1}{n !} \frac{\partial \hat{\theta}(\eta ; \zeta)}{\partial \eta} \\
& \hat{\phi}_{n}(\eta)=\frac{1}{n !} \frac{\partial \hat{\phi}(\eta ; \zeta)}{\partial \eta} .
\end{aligned}
$$

There are BCs as

$$
\begin{gathered}
\hat{f}(0)=0, \\
\hat{f}^{\prime}(0)=1, \\
\hat{\theta}(0)=1, \\
\hat{\phi}(0)=1, \\
\hat{f}^{\prime}(\infty)=A, \\
\hat{\theta}(\infty)=0, \\
\hat{\phi}(\infty)=0 .
\end{gathered}
$$

$$
\begin{aligned}
& \mathfrak{R}_{n} \widehat{f}(\eta)=\left(1-\widehat{\phi}+\widehat{\phi} \frac{\rho_{s}}{\rho_{f}}\right)(1-\widehat{\phi})^{2.5} A^{2}+(1+2 \gamma \eta)-f^{\prime}+2 \gamma \widehat{f}^{\prime \prime}+\left(1-\widehat{\phi}+\widehat{\phi} \frac{\rho_{s}}{\rho_{f}}\right) \\
& \times \mathfrak{R}_{n} \hat{\theta}(\eta)=(2 \gamma \eta+1)\left[\frac{k_{n f}}{k_{f}}\left(1+(N r-1) \hat{\theta}_{n-1}\right)^{3}\right] \hat{\theta}_{n-1}^{\prime \prime}+\left[\frac{1}{2 k^{*}} \frac{k_{n f}}{k_{f}}\left(1+(N r-1) \hat{\theta}_{n-1}\right)^{3}\right] \gamma \widehat{\theta}_{n-1}^{\prime} \\
& +\frac{\operatorname{Pr}}{4 k^{*}}\left[1-\phi+\phi \frac{\left(\rho_{c p}\right)_{s}}{\left(\rho_{c p}\right)_{f}}\right] \sum_{j=0}^{w-1} \hat{\theta}_{w-1-j}^{\prime} \hat{f}_{j}^{\prime}-\hat{f}^{\prime} \hat{\theta}_{n-1}+D c \bar{\theta}_{n-1}+\frac{E c}{(1-\phi)^{2.5}} \hat{f}^{\prime^{2}} \\
& +2(1+2 \gamma \eta)(N r-1)\left(1+(N r-1) \widehat{\theta}_{n-1}\right)^{2} \hat{f}^{\prime^{2}}{ }_{n-1}+\operatorname{Pr}(1+2 \gamma \eta) N b \sum_{j=0}^{w-1} \hat{\phi}_{w-1-j}^{\prime}{\bar{\theta}_{j}^{\prime}}_{j}+N t{\bar{\theta}^{\prime 2}}^{2} \\
& \mathfrak{R}_{n} \widehat{\phi}_{n}(\eta)=(2 \gamma \eta+1) \widehat{\phi}_{n-1}^{\prime \prime}+2 \gamma \widehat{\phi}_{n-1}^{\prime}+S c \sum_{j=0}^{w-1} \widehat{f}_{w-1-j}{\widehat{\phi^{\prime}}}_{j}+\frac{N t}{N b}\left[(1+2 \gamma \eta) \widehat{\theta}_{n-1}^{\prime}+2 \gamma \widehat{\theta}_{n-1}^{\prime}\right]+\operatorname{Prle\varepsilon } \widehat{\phi}_{n-1} \\
& (1-\widehat{\phi})^{2.5} \sum_{j=0}^{w-1}\left(\hat{f}_{w-1-j} \hat{f}_{j}^{\prime \prime}-\hat{f}^{\prime 2}\right)-(1-\widehat{\phi})^{2.5} M-f^{\prime} .
\end{aligned}
$$


Since HAM technique is the iterative process, the number of iterations is more important for the convergence of problem solution, so we are selecting a general order solution, that is, $j^{\text {th }}$-order solution. Symbolically it can be written as

$$
\left.\begin{array}{l}
N_{\widehat{f}}\left[\hat{f}_{j}(\eta)-\chi_{j} \hat{f}_{j-1}(\eta)\right]=\hbar_{f} \mathfrak{R}_{j}^{\hat{f}}(\eta), \\
N_{\hat{\theta}}\left[\hat{\theta}_{j}(\eta)-\chi_{j} \hat{\theta}_{j-1}(\eta)\right]=\hbar_{-} \mathfrak{R}_{j}^{\hat{\theta}}(\eta), \\
N_{\widehat{\phi}}\left[\hat{\phi}_{j}(\eta)-\chi_{j} \hat{\phi}_{j-1}(\eta)\right]=\hbar_{-} \mathfrak{R}_{j}^{\bar{\phi}}(\eta) .
\end{array}\right\}
$$$$
\text { Here, } \chi_{n}=\left\{\begin{array}{ll}
0, & \text { if } \zeta \leq 1 \\
1, & \text { if } \zeta>1
\end{array}\right. \text {. }
$$

\section{Results and Discussion}

In this section, the impact of $\phi, M, A, S_{1}$ and $\gamma$ on $f^{\prime}(\eta)$ is discussed in detail with practical applications which are presented graphically in Figures 2-6. Here, Figure 2 displays the impact of volume fraction $\varphi$ of the nanoparticles on velocity profile $f^{\prime}(\eta)$. It is seen from Figure 2 that, by increasing the value of $\varphi$, the axial velocity $f^{\prime}(\eta)$ is increasing. Physically the increase in nanoparticles volume fraction in base fluid means gain of energy so the nanofluid with more energy moves with high speed. Figure 3 shows that if we increase the value of $M$ the velocity profile is going to decay. Physically, the very strong magnetic field is causing an increase in the Lorentz force; hence, the more opposing force makes it difficult for the fluid to flow easily. Figure 4 displays the effect of velocity ratio parameter $A$ on $f^{\prime}(\eta)$ in the presence of slip parameter. It is observed that if the value of $A$ is increasing, the axial velocity $f^{\prime}(\eta)$ enhances. Physically, $A=U_{\infty} / U_{0}<1$ means that the free stream velocity is less than the stretching velocity of the cylinder, and with this difference in velocities a boundary layer structure is formed which goes to decrease in motion very close to stagnation point and as a result this leads to increase in the boundary layer as $A$ increases. Figure 5 shows that by increasing the value of $S_{1}$ (velocity slip parameter) the velocity profile $f^{\prime}(\eta)$ reduces. Physically, this phenomenon occurs due to the fact that the stretching velocity up to some extent shifts to the fluid and thus a decrease behavior is shown in the velocity profile. Figure 6 shows that $f^{\prime}(\eta)$ is an increasing function of $\gamma$. Physically, as curvature parameter $\gamma$ increases the radius of the cylinder decreases; then in fact the area between the fluid and the cylinder becomes lesser as the radius of the cylinder becomes short; hence the increasing behavior in velocity profile $f^{\prime}(\eta)$ of the fluid is seen when we increase the value of $\gamma$. The impacts of several convergence physical parameters on velocity profiles $f(\eta)$ are shown in Figures 7-11. The different physical parameters for velocity profiles $f(\eta)$ are the ratio of velocities $A$, the curvature parameter $\gamma$, magnetic parameter $M$, velocity slip parameter $S_{1}$, and volume fraction $\varphi$. It is seen in Figure 7 that by the cumulative value of ratio velocity $A$ the enhancement behavior in the momentum profile is seen. Figure 8 demonstrates that if the value of curvature parameter increases then the reduction in momentum profile is observed. Figure 9

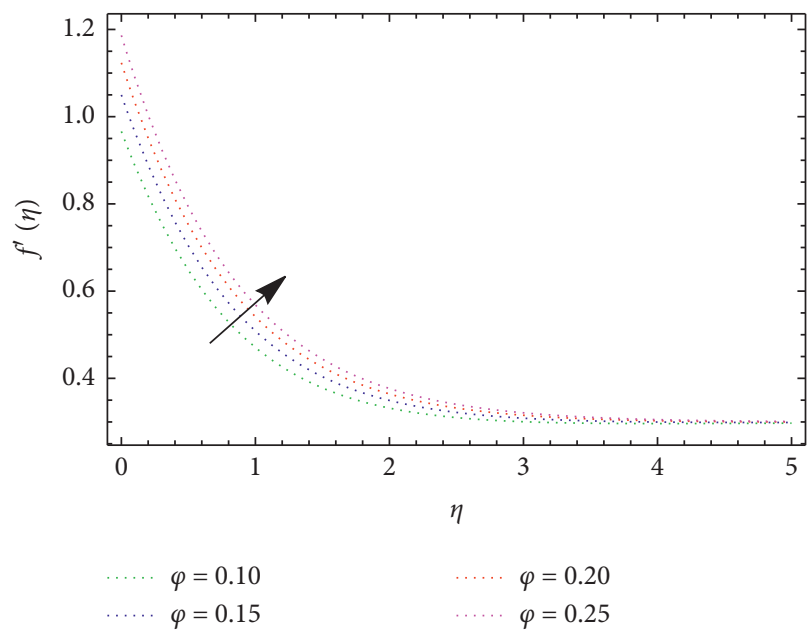

Figure 2: Axial velocity profile for different values of $\varphi$.

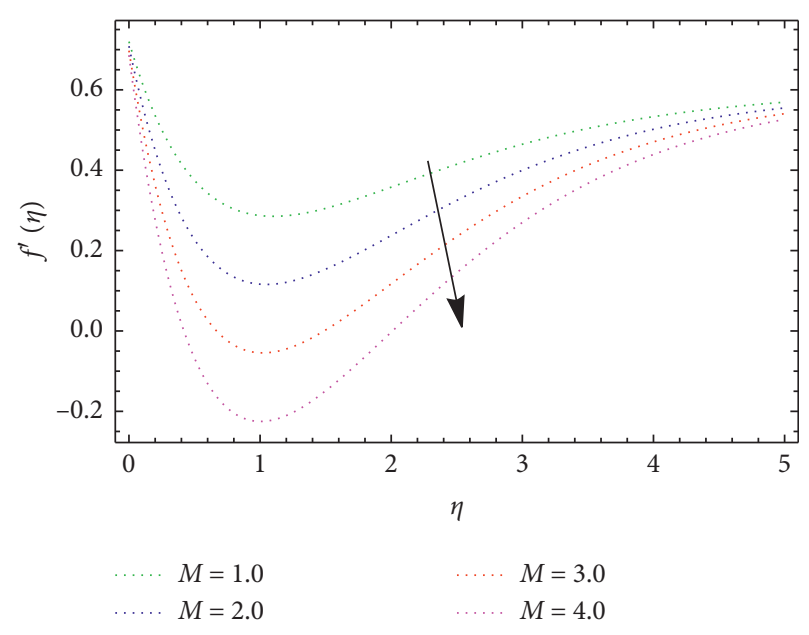

FIgURE 3: Axial velocity profile for the alternate values of $M$.

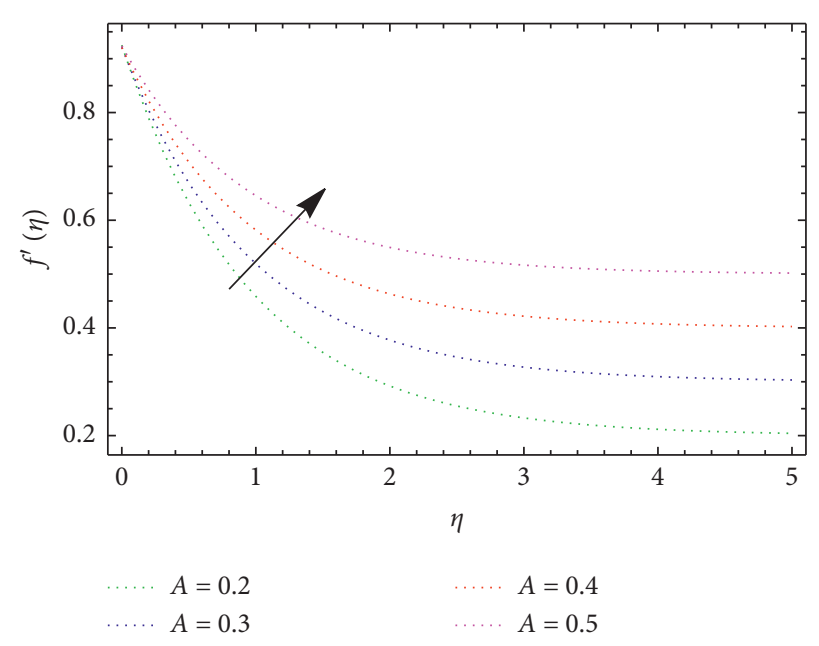

Figure 4: The profile of velocity for the numerous values of $A$. 


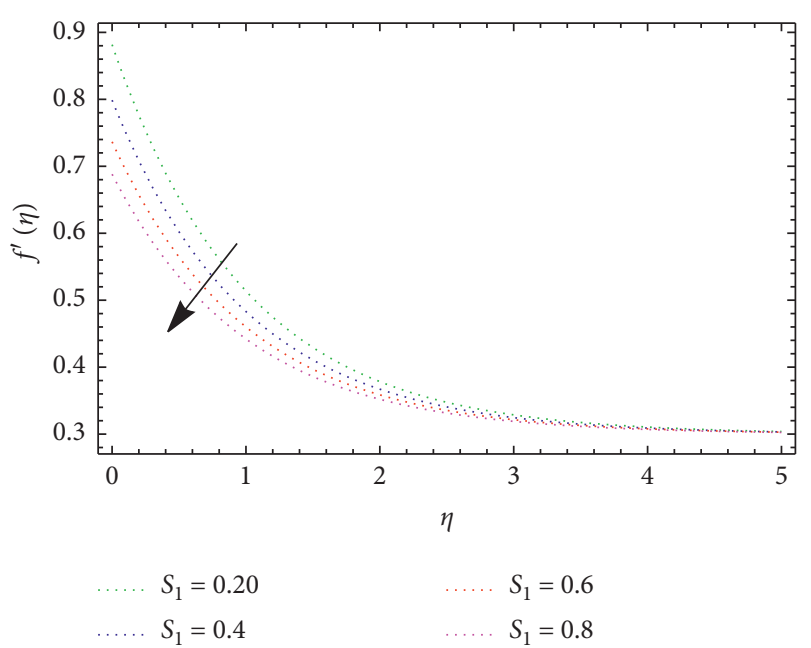

Figure 5: Impact of $S_{1}$ on velocity profile $f^{\prime}(\eta)$.

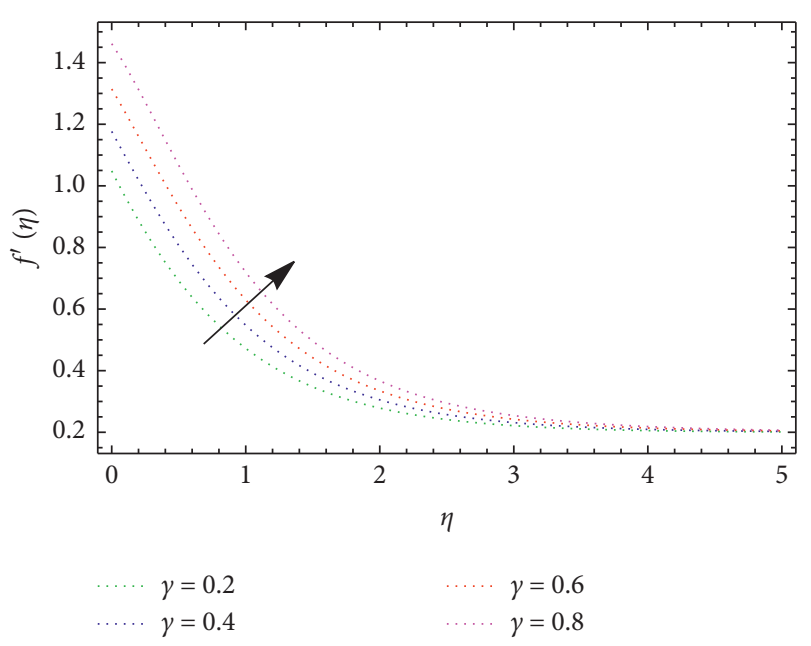

Figure 6: The velocity profile $f^{\prime}(\eta)$ for various values of $\gamma$.

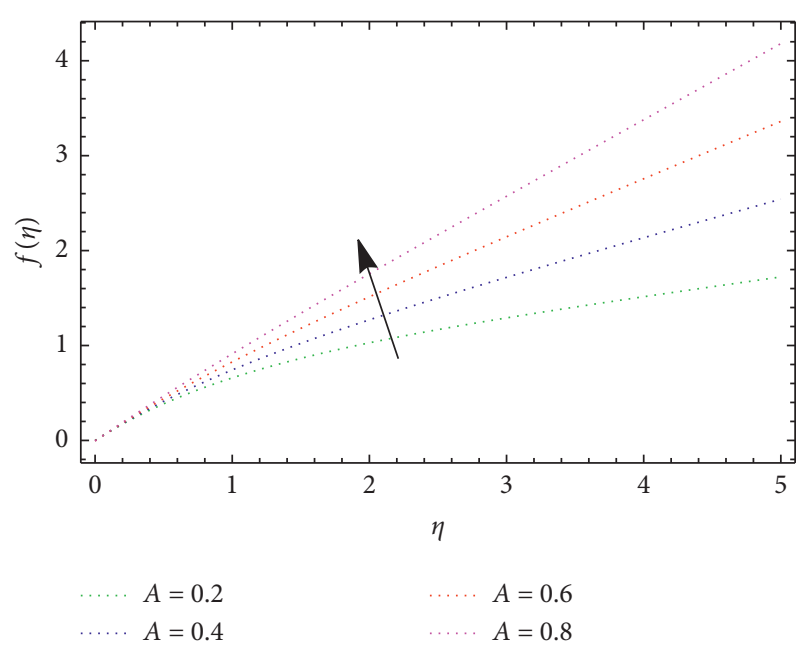

Figure 7: Effects of $A$ on $f(\eta)$.

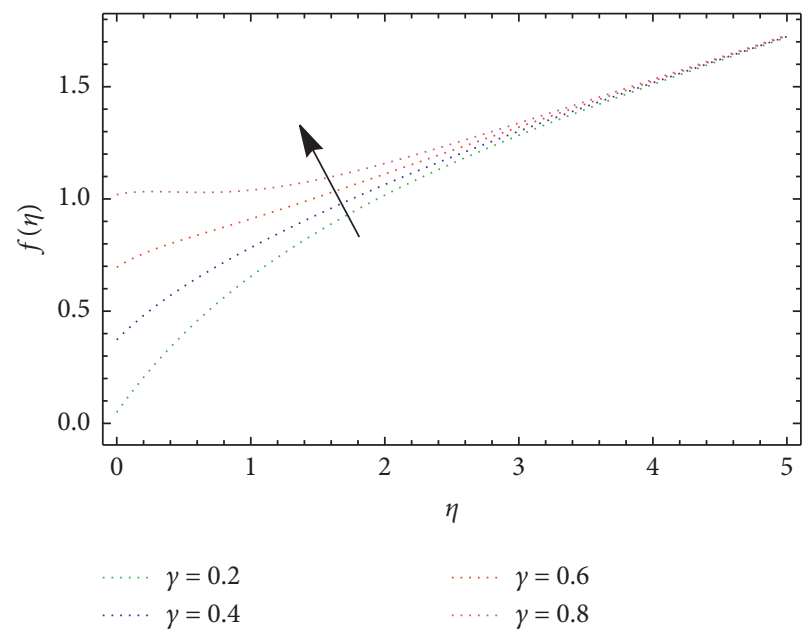

Figure 8: Effects of $\gamma$ on $f(\eta)$.

shows the behavior of magnetic parameter on velocity profile $f(\eta)$ by growing the value of magnetic parameter and then the momentum profile shows the reduction in its behavior. Figure 10 shows that by the increase in velocity slip parameter $S_{1}$ the reduction is seen in the behavior of momentum profile $f(\eta)$. Figure 11 demonstrates the impact of nanoparticles volume fraction $\varphi$ on the momentum profile $f(\eta)$ of the flow. In Figure 11 it is observed that with the augmentation in nanoparticle fraction in the base fluid the pace $f(\eta)$ of the nanofluid reduces. Physically the augmentation of the nanoparticle volume fraction in the base fluid goes to the augmentation of the viscosity of the nanofluid and it is obvious that more viscous fluid flows with low pace. Figures 12-15 analyze the effect of different physical parameters $\gamma, M, D c$, and Econ temperature profile $\theta(\eta)$. Figure 12 shows the effect of curvature parameter $\gamma$ on temperature field $\theta(\eta)$. The fluid temperature boosts for the augmentation in the values of curvature parameter $\gamma$ of the cylinder. Physically, this is due to the fact that by increasing $\gamma$ which means a decrease in radius of the cylinder this goes to decrease the gap among fluid and cylinder; it increases the heat transport and thus raises temperature. Figure 13 shows the impact of $M$ on the temperature profile $\theta(\eta)$. It is obvious that by increasing value of $M$ the temperature profile increases. Physically, the increase in magnetic parameter Mreduces the velocity profile of the fluid; hence, low moving fluid gains more energy due to large interval of time in contact with hot cylinder. Figures 14 and 15 demonstrate the impact of heat generation parameter $D c$ and Eckert number $E c$ on temperature profile $\theta(\eta)$. It is noted from Figure 14 that by the enhancement of heat generation parameter $D c$ the temperature profile $\theta(\eta)$ of the nanofluid enhances. Figure 15 demonstrates the impact of Eckert number $E c$ on the temperature profile $\theta(\eta)$ of the nanofluid. From Figure 15 it is obvious that by the augmentation of Eckert number $E c$ the temperature profile $\theta(\eta)$ of the nanofluid enhances. Physically, enhancement of Eckert number is the increase in kinetic energy of the fluid due to which temperature profile $\theta(\eta)$ of the fluid enhances. Figure 16 demonstrates the impact of thermal radiation 


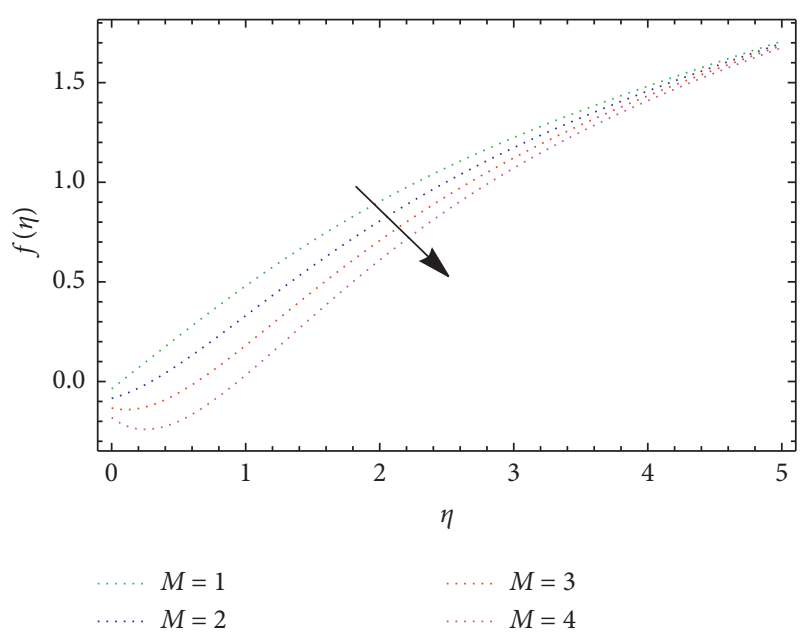

Figure 9: Effects of $M$ on $f(\eta)$.

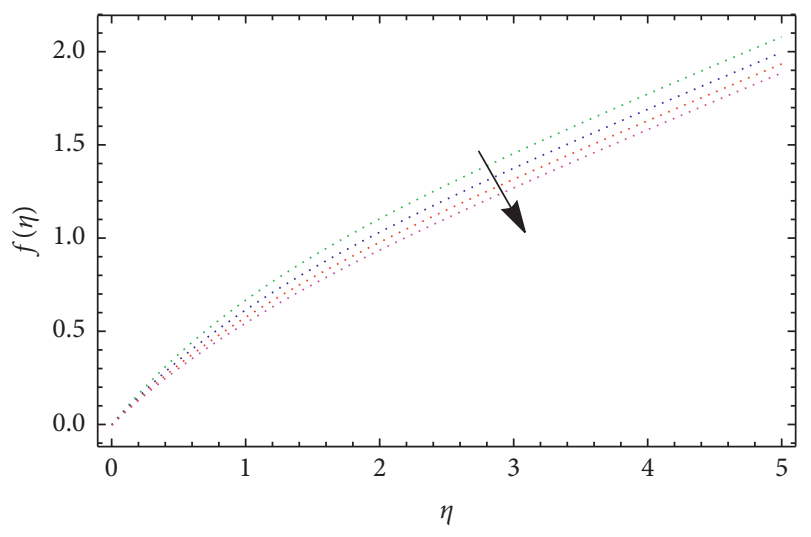
$S_{1}=0.2$
$S_{1}=0.4$

$$
S_{1}=0.6
$$$$
S_{1}=0.8
$$

Figure 10: Effects of $S_{1}$ on $f(\eta)$.

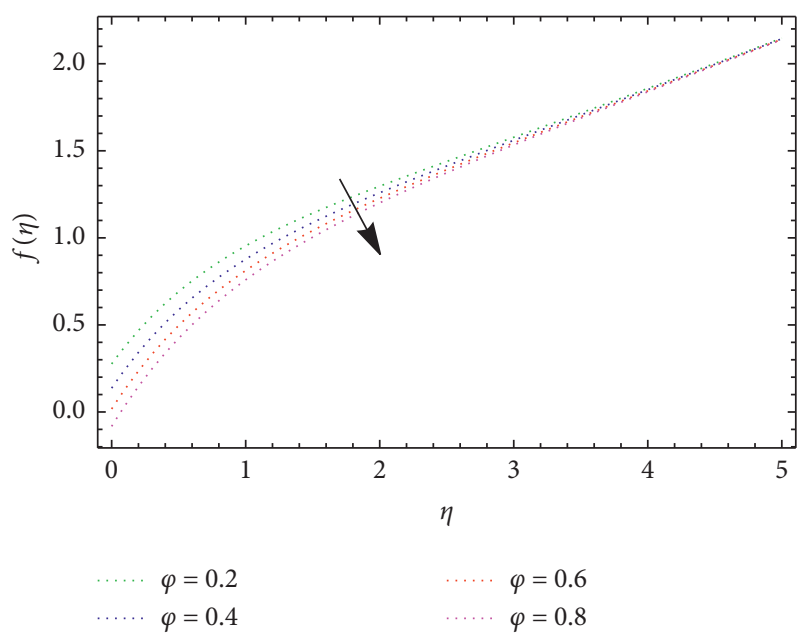

Figure 11: Effects of $\varphi$ on $f(\eta)$.

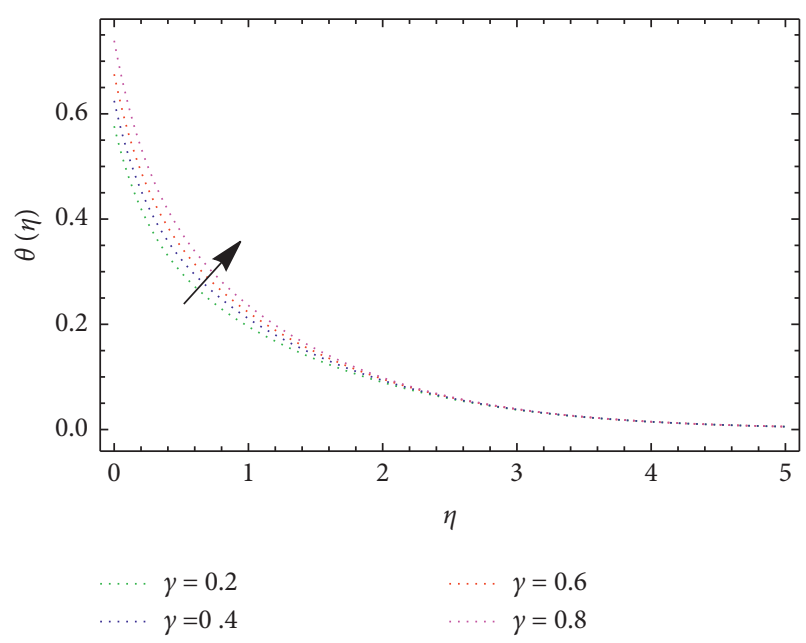

Figure 12: Effects of $\gamma$ on $\theta(\eta)$.

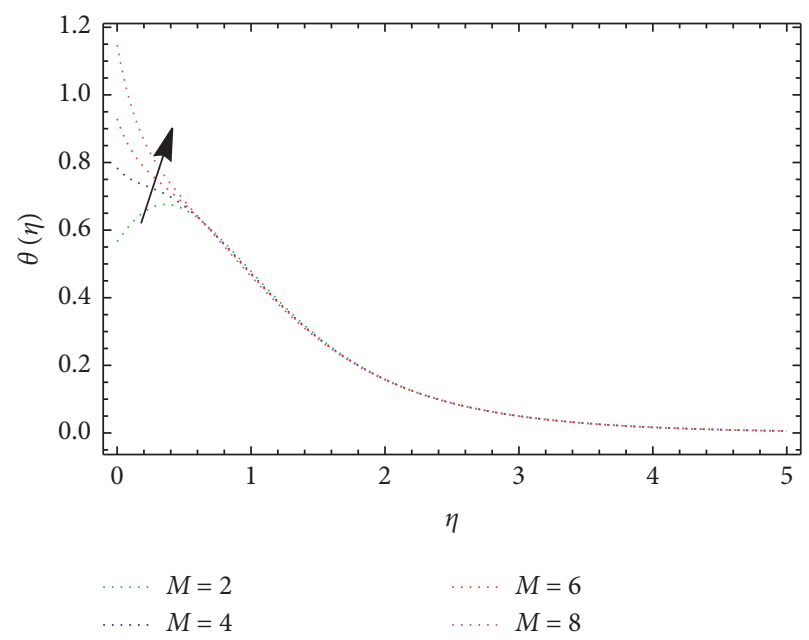

Figure 13: Effects of $M$ on $\theta(\eta)$.

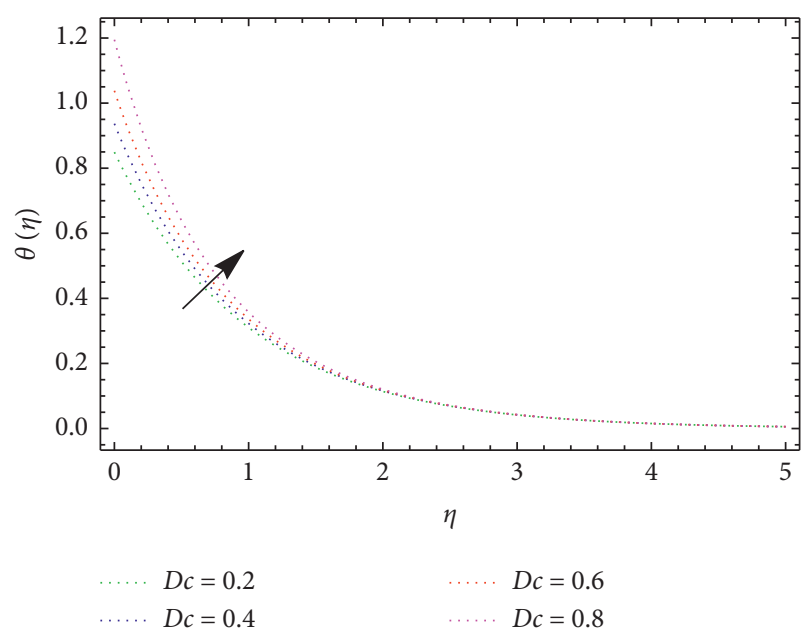

Figure 14: Effects of $D$ con $\theta(\eta)$. 


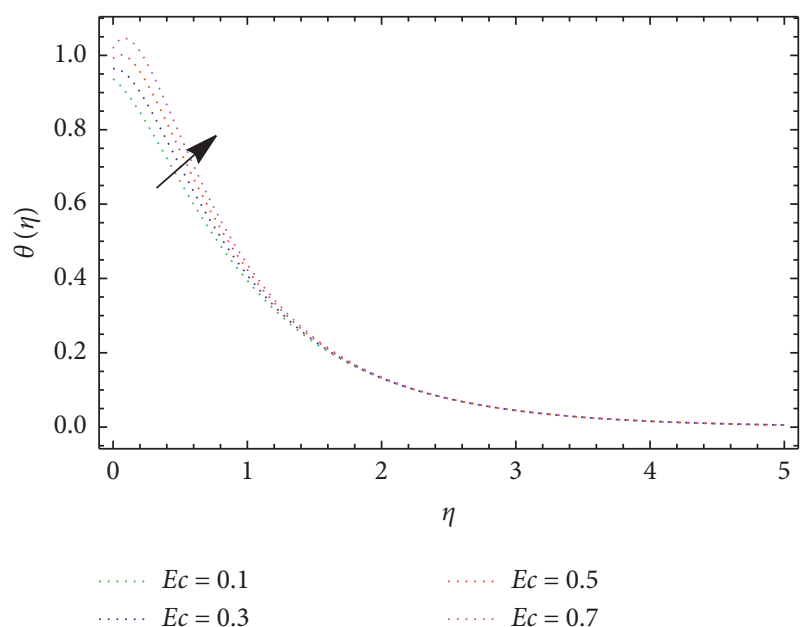

Figure 15: Effects of $E c$ on $\theta(\eta)$.

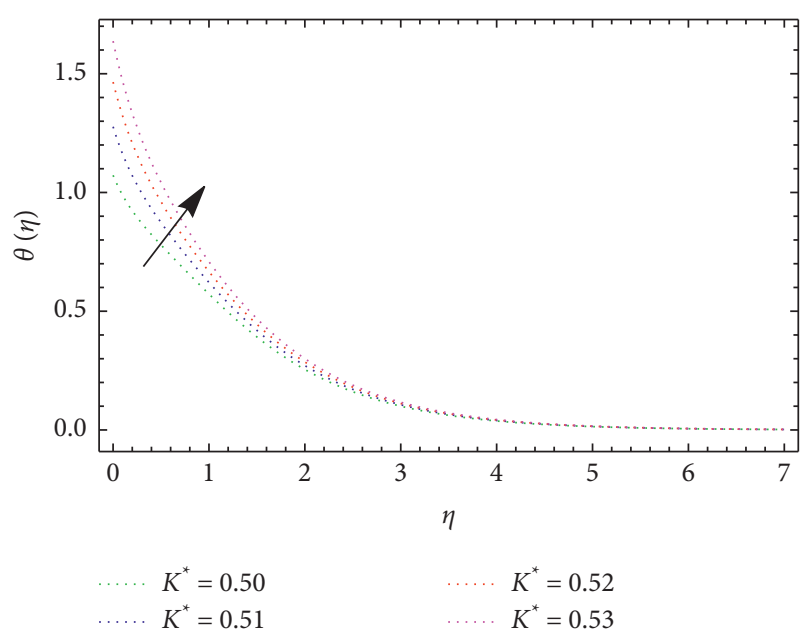

Figure 16: Effects of $K^{*}$ on $\theta(\eta)$.

parameter $K^{*}$ on the temperature profile of the nanofluid; it is very clear from this figure that the temperature profile $\theta(\eta)$ enhances with the escalation of thermal radiation parameter $K^{*}$. Figure 17 is drawn to observe the influence of $N t$ on $\theta(\eta)$. It is noticed that by increasing the value of the thermophoretic parameter $N t$ there is an increase in concentration profile $\theta(\eta)$; by this we mean that too many nanoparticles go away from the hot surface and thus the volume fraction distribution boosts. Figure 18 shows the impact of Schmidt number Sc on $\phi(\eta)$. By increasing the value of $S c$, the concentration profile $\theta(\eta)$ decreases. Physically, by increasing value of $S c$ the Brownian diffusion coefficient rises and also the thickness of boundary layer shows a decreasing result. Figure 19 shows the impact of $\mathrm{Nb}$ on $\theta(\eta)$. It is observed that by increasing the value of $N b$ the zigzag movement and also the colliding of macroscopic particles of the fluid increase; as a result $\theta(\eta)$ decreases. Figure 20 demonstrates the impact of chemical reaction on the concentration profile of the nanofluid. In Figure 20 it is

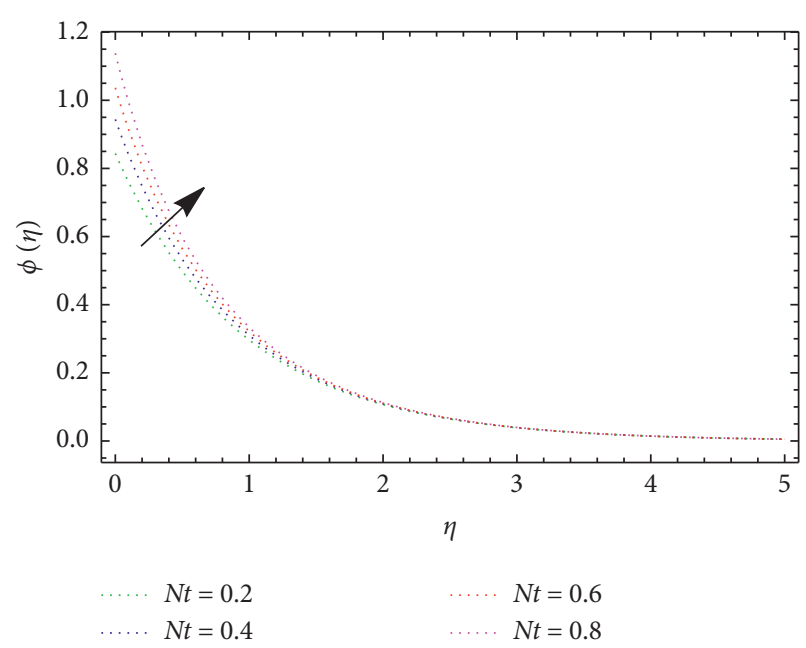

Figure 17: Effects of $N t$ on $\phi(\eta)$.

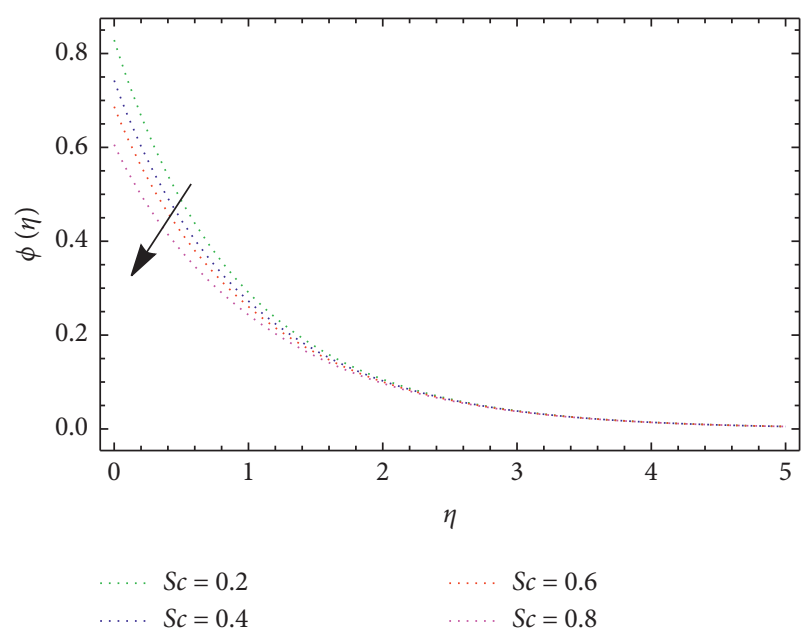

Figure 18: Effects of $S c$ on $\phi(\eta)$.

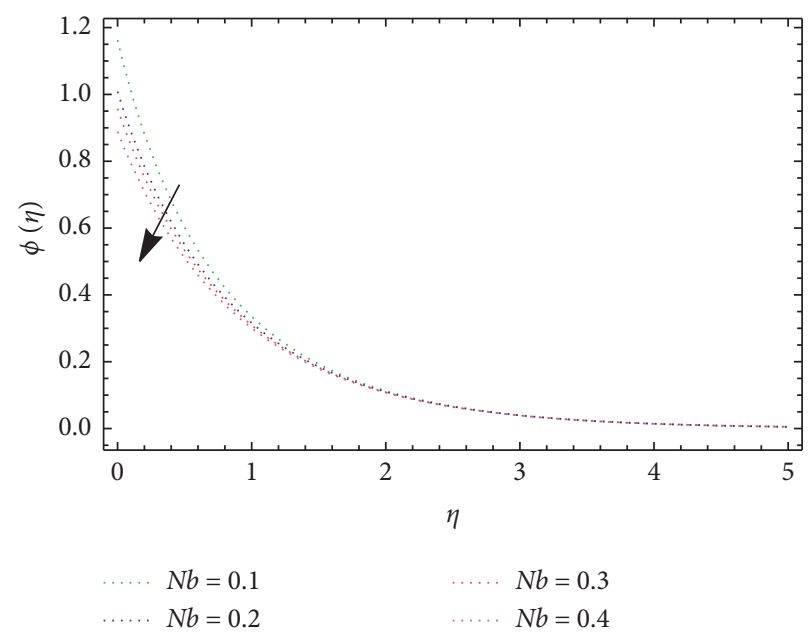

Figure 19: Effects of $\mathrm{Nb}$ on $\phi(\eta)$. 


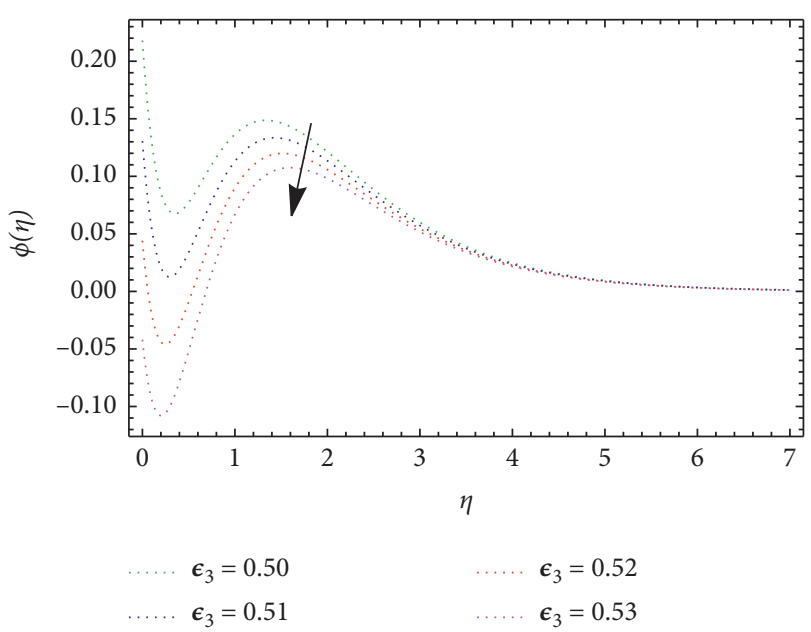

Figure 20: Effects of $\epsilon_{3}$ on $\phi(\eta)$.

TABLe 2: Comparison of present and previous results for Nusselt number $\left(N u_{x} \mathrm{Re}_{x}^{-1 / 2}\right)$ at $\mathrm{Pr}=10$.

\begin{tabular}{ccccccc}
\hline$\gamma$ & $\phi$ & $M$ & $N r$ & $K^{*}$ & $\begin{array}{c}\text { Suleman et al. [39] } \\
N u_{x} \mathrm{Re}_{x}^{-1 / 2}\end{array}$ & $\begin{array}{c}\text { Present result } \\
N u_{x} \mathrm{Re}_{x}^{-1 / 2}\end{array}$ \\
\hline 1.0 & 0.3 & 1.0 & 0.1 & 2.0 & 0.93109 & 0.93110 \\
2.0 & & & & & 0.99247 & 0.99249 \\
3.0 & & & & & 1.03770 & 1.03773 \\
& 0.1 & & & & 0.68440 & 0.68444 \\
& 0.2 & & & & 0.79711 & 0.79714 \\
& 0.3 & & & & 0.93109 & 0.93110 \\
& 1.0 & & & 0.93109 & 0.93111 \\
& 2.0 & & & 0.92688 & 0.92690 \\
& 3.0 & & & 0.92304 & 0.92306 \\
& & 0.1 & & 0.93109 & 0.93110 \\
& & 0.2 & & 0.87770 & 0.87773 \\
& & 0.3 & & 0.42070 & 0.42073 \\
& & & 1.0 & 0.97827 & 0.97829 \\
& & & 2.0 & 0.93109 & 0.93110 \\
& & & 3.0 & 0.88391 & 0.88393 \\
\hline
\end{tabular}

observed that with the boosting of chemical reaction parameter $\epsilon_{3}$ the concentration profile decreases. Physically, the chemical reaction $\epsilon_{3}<0$ indicates the nondestructive reaction, that is, the binding of nanoparticles, which results in the decrease in concentration of the fluid and as a result the decrease in thickness of boundary layer. Table 1 shows thermophysical properties of silver nanoparticles and water used in this article's base fluid. Table 2 shows the comparison of heat transfer rate during the current phenomenon with previous result. Table 3 presents the impact of various physical parameters on the mass transfer past a stretched cylinder under the impact of MHD. Table 4 shows the impact of various physical parameters on the heat transfer rate during the nanofluid flow past a stretched cylinder. Table 5 displays the impact of various physical parameters on the skin friction between the nanofluid and surface of the stretched cylinder.
TABle 3: Sherwood number $\left(S h_{x} \operatorname{Re}_{x}^{-/ 1 / 2}\right)$ for $S c, N b, N t$, and $\operatorname{Pr}$ when $S_{1}=0.5$.

\begin{tabular}{cccccc}
\hline$S c$ & $N b$ & $N t$ & $\mathrm{Pr}$ & $\epsilon_{3}$ & $S h_{x} \mathrm{Re}_{x}^{-1 / 2}$ \\
\hline 0.6 & 0.1 & 0.1 & 1.0 & -0.5 & 0.844887 \\
0.8 & & & & & 0.836159 \\
0.9 & & & & & 0.831806 \\
& 0.2 & & & & 0.918544 \\
& 0.4 & & & 0.863301 \\
& 0.6 & & & & 0.844887 \\
& & 0.1 & & & 0.862905 \\
& 0.2 & & & 0.918143 \\
& 0.4 & & & 0.831806 \\
& & & & & 0.832831 \\
& & & & & 0.833855 \\
& & & & -0.5 & 0.597832 \\
& & & & 0.2 & 0.586579 \\
& & & & & 0.567842 \\
\hline
\end{tabular}

TABLE 4: Numerical value for heat transfer rate $\left(N u_{x} \operatorname{Re}_{x}^{-1 / 2}\right)$ for $\gamma$, $E c$, and $D c$ when $\operatorname{Pr}=3$.

\begin{tabular}{ccccc}
\hline$M$ & $\gamma$ & $E c$ & $D c$ & $N u_{x} \mathrm{Re}_{x}^{-1 / 2}$ \\
\hline 1 & 0.2 & 0.2 & 0.1 & 0.180844 \\
2 & & & & 0.157272 \\
3 & 0.1 & & & 0.133700 \\
& 0.2 & & & 0.083679 \\
& 0.3 & & & 0.133700 \\
& & 0.1 & & 0.184026 \\
& & 0.2 & & 0.695915 \\
& 0.3 & & 0.745186 \\
& & & 0.2 & 0.794457 \\
& & & 0.3 & 0.7455186 \\
\hline
\end{tabular}

TABLE 5: Value of skin friction for the given involved parameters.

\begin{tabular}{ccccc}
\hline$M$ & $\gamma$ & $\phi$ & $A$ & $C_{f} \operatorname{Re}_{x}^{1 / 2}$ \\
\hline 1.0 & 1.0 & 0.3 & 0.2 & 1.60240 \\
2.0 & & & 2.10020 \\
3.0 & & & 2.97470 \\
& 1.0 & & & 2.10020 \\
& 2.0 & & & 2.55200 \\
& 3.0 & 0.1 & & 2.96720 \\
& & 0.2 & & 1.11380 \\
& 0.3 & & 1.93740 \\
& & & 0.0 & 2.10020 \\
& & & 0.5 & 0.84040 \\
& & & 0.7 & 0.294600 \\
& & &
\end{tabular}

\section{Conclusions}

The current exploration deals with the study of nanofluid, which is a mixture of nanoparticles (silver) and a base fluid (water), as well as nonlinear thermal radiation and Newtonian heating as it passes through a nonlinear stretching cylinder. The coupled nonlinear differential equations are 
solved using the homotopy analysis method (HAM). As a consequence, we arrive to the following conclusions:

(i) For the larger value of $S>0$, the velocity distribution $f^{\prime}(\eta)$ will reduce.

(ii) For the increasing value of solid volume fraction of the nanoparticles, the increase of the velocity field is detected.

(iii) The velocity and temperature profiles show contrasting result for the magnetic parameter. The velocity profile is shrinking and temperature profile becomes enlarged.

(iv) For the increasing value of curvature and radiation parameters, the temperature profile boosts.

(v) Sherwood number $\left(S h_{x} \mathrm{Re}_{x}^{-1 / 2}\right)$ has opposite effects for $N t$ and $N b$.

\section{Data Availability}

The data used to support the findings of this study are included within the article.

\section{Conflicts of Interest}

The authors declare that they have no conflicts of interest.

\section{Acknowledgments}

The authors extend their appreciation to the Deanship of Scientific Research at King Khalid University, Abha, Saudi Arabia, for funding this work through research groups program under Grant no. R.G.P-1/128/42.

\section{References}

[1] S. U. S. Choi, "Nanofluids: a new field of scientific research and innovative applications," Heat Transfer Engineering, vol. 29, no. 5, pp. 429-431, 2008.

[2] D. Pal, G. Mandal, and K. Vajravalu, "Soret and Dufour effects on MHD convective-radiative heat and mass transfer of nanofluids over a vertical non-linear stretching/shrinking sheet," Applied Mathematics and Computation, vol. 287-288, pp. 184-200, 2016.

[3] T. Hussain, S. A. Shehzad, T. Hayat, A. Alsaedi, F. Al-Solamy, and M. Ramzan, "Radiative hydromagnetic flow of Jeffrey nanofluid by an exponentially stretching sheet," Plos One, vol. 9, no. 8, Article ID e103719, 2014.

[4] K. Bhattacharyya and G. C. Layek, "Magnetohydrodynamic boundary layer flow of nanofluid over an exponentially stretching permeable sheet," Physics Research International, vol. 2014, Article ID 592536, 12 pages, 2014.

[5] M. M. Rashidi, S. Abelman, and N. Freidooni Mehr, "Entropy generation in steady MHD flow due to a rotating porous disk in a nanofluid," International Journal of Heat and Mass Transfer, vol. 62, pp. 515-525, 2013.

[6] F. Mabood and A. Mastroberardino, "Melting heat transfer on MHD convective flow of a nanofluid over a stretching sheet with viscous dissipation and second order slip," Journal of the Taiwan Institute of Chemical Engineers, vol. 57, pp. 62-68, 2015.
[7] T. Hayat, M. Waqas, S. A. Shehzad, and A. Alsaedi, "A model of solar radiation and Joule heating in magnetohydrodynamic (MHD) convective flow of thixotropic nanofluid," Journal of Molecular Liquids, vol. 2015, pp. 704-710, 2016.

[8] A. Shafiq, G. Rasool, H. Alotaibi et al., "Thermally enhanced Darcy-Forchheimer Casson-water/glycerine rotating nanofluid flow with uniform magnetic field," Micromachines, vol. 12 , no. 6 , p. 605,2021

[9] G. Rasool and A. Shafiq, "Numerical exploration of the features of thermally enhanced chemically reactive radiative Powell-Eyring nanofluid flow via Darcy medium over nonlinearly stretching surface affected by a transverse magnetic field and convective boundary conditions," Applied Nanoscience, pp. 1-18, 2020.

[10] H. Alotaibi, S. Althubiti, M. Eid, and K. Mahny, "Numerical treatment of mhd flow of casson nanofluid via convectively heated non-linear extending surface with viscous dissipation and suction/injection effects," Computers, Materials \& Continua, vol. 66, no. 1, pp. 229-245, 2020.

[11] N. Y. Abd Elazem and Y. Nader, "Numerical results for influence the flow of MHD nanofluids on heat and mass transfer past a stretched surface," Nonlinear Engineering, vol. 10, no. 1, pp. 28-38, 2021.

[12] T. Muhammad, A. Alsaedi, S. A. Shehzad, and T. Hayat, "A revised model for Darcy-Forchheimer flow of Maxwell nanofluid subject to convective boundary condition," Chinese Journal of Physics, vol. 55, no. 3, pp. 963-976, 2017.

[13] S. J. Liao, Homotopy Analysis Method in Nonlinear Differential Equations, Springer and Higher Education Press, Heidelberg, Germany, 2012.

[14] T. Hayat, A. Nassem, M. I. Khan, M. Farooq, and A. Al-Saedi, "Magnetohydrodynamic (MHD) flow of nanofluid with double stratification and slip conditions," Physics and Chemistry of Liquids, vol. 56, no. 2, pp. 189-208, 2018.

[15] H. Waqas, M. Hussain, M. S. Alqarni, M. R. Eid, and T. Muhammad, "Numerical simulation for magnetic dipole in bioconvection flow of Jeffrey nanofluid with swimming motile microorganisms," Waves in Random and Complex Media, pp. 1-18, 2021.

[16] M. Khan, J. Ahmed, and W. Ali, "Thermal analysis for radiative flow of magnetized Maxwell fluid over a vertically moving rotating disk," Journal of Thermal Analysis and Calorimetry, vol. 143, no. 6, pp. 4081-4094, 2021.

[17] G. K. Ramesh, S. A. Shehzad, A. Rauf, and A. J. Chamkha, "Heat transport analysis of aluminum alloy and magnetite graphene oxide through permeable cylinder with heat source/ sink," Physica Scripta, vol. 95, no. 9, Article ID 095203, 2020.

[18] M. Ramzan, N. Gul, J. D. Chung, S. Kadry, and Y. M. Chu, "“Numerical treatment of radiative Nickel-Zinc ferrite-Ethylene glycol nanofluid flow past a curved surface with thermal stratification and slip conditions," Scientific Reports, vol. 10, no. 1, pp. 1-14, 2020.

[19] D. U. Sarwe and V. S. Kulkarni, “Thermal behaviour of annular hyperbolic fin with temperature dependent thermal conductivity by differential transformation method and Pade approximant," Physica Scripta, vol. 96, no. 10, Article ID 105213, 2021.

[20] K. Hosseinzadeh, M. Gholinia, B. Jafari, A. Ghanbarpour, H. Olfian, and D. D. Ganji, "Nonlinear thermal radiation and chemical reaction effects on Maxwell fluid flow with convectively heated plate in a porous medium," Heat Transfer Asian Research, vol. 48, no. 2, pp. 744-759, 2019.

[21] R. N. Kumar, R. J. P. Gowda, J. K. Madhukesh, B. C. Prasannakumara, and G. K. Ramesh, "Impact of 
thermophoretic particle deposition on heat and mass transfer across the dynamics of Casson fluid flow over a moving thin needle," Physica Scripta, vol. 96, no. 7, Article ID 075210, 2021.

[22] R. N. Kumar, R. J. P. Gowda, G. D. Prasanna, B. C. Prasannakumara, K. S. Nisar, and W. Jamshed, "Comprehensive study of thermophoretic diffusion deposition velocity effect on heat and mass transfer of ferromagnetic fluid flow along a stretching cylinder," Proceedings of the Institution of Mechanical Engineers - Part E: Journal of Process Mechanical Engineering, Article ID 09544089211005291, 2021.

[23] S. M. Venthan, I. Jayakaran Amalraj, P. Senthil Kumar, and M. S. Nisha, "Non-Newtonian nanofluids flow analysis at the ingress section in process intensified system," Chemical Engineering and Processing - Process Intensification, vol. 167, Article ID 108518, 2021.

[24] A. Rehman, A. Hussain, and S. Nadeem, "Physical aspects of convective and radiative molecular theory of liquid originated nanofluid flow in the existence of variable properties," Physica Scripta, vol. 96, no. 3, Article ID 035219, 2021.

[25] M. Ramzan, "Influence of Newtonian heating on three dimensional MHD flow of couple stress nanofluid with viscous dissipation and joule heating," PloS One, vol. 10, no. 4, Article ID e0124699, 2015.

[26] S. Qayyum, T. Hayat, S. A. Shehzad, and A. Alsaedi, "Mixed convection and heat generation/absorption aspects in MHD flow of tangent-hyperbolic nanoliquid with Newtonian heat/ mass transfer," Radiation Physics and Chemistry, vol. 144, pp. 396-404, 2018.

[27] M. K. A. Mohamed, M. Z. Salleh, N. A. Z. Noar, and A. Ishak, "Buoyancy effect on stagnation point flow past a stretching vertical surface with Newtonian heating," AIP Conference Proceedings, vol. 1795, no. 1, Article ID 020005, 2017.

[28] N. S. Akbar and S. Nadeem, "Mixed convective magnetohydrodynamic peristaltic flow of a Jeffrey nanofluid with Newtonian heating," Zeitschrift für Naturforschung A, vol. 68, no. 6, pp. 433-441, 2013.

[29] O. D. Makinde, "Computational modelling of MHD unsteady flow and heat transfer toward a flat plate with Navier slip and Newtonian heating," Brazilian Journal of Chemical Engineering, vol. 29, no. 1, pp. 159-166, 2012.

[30] M. U. Rahman, M. Khan, and M. Manzur, "Homogeneousheterogeneous reactions in modified second grade fluid over a non-linear stretching sheet with Newtonian heating," Results in Physics, vol. 7, pp. 4364-4370, 2017.

[31] I. Khan, M. Y. Malik, T. Salahuddin, M. Khan, and K. U. Rehman, "Homogenous-heterogeneous reactions in MHD flow of Powell-Eyring fluid over a stretching sheet with Newtonian heating," Neural Computing \& Applications, vol. 30, no. 11, pp. 3581-3588, 2017.

[32] N. Acharya, R. Bag, and P. K. Kundu, "Unsteady bioconvective squeezing flow with higher-order chemical reaction and second-order slip effects," Heat Transfer, 2021.

[33] N. Acharya, "Spectral simulation to investigate the effects of active passive controls of nanoparticles on the radiative nanofluidic transport over a spinning disk," Journal of Thermal Science and Engineering Applications, vol. 13, no. 3, Article ID 031023, 2021.

[34] N. Acharya, "On the flow patterns and thermal behaviour of hybrid nanofluid flow inside a microchannel in presence of radiative solar energy," Journal of Thermal Analysis and Calorimetry, vol. 141, no. 4, pp. 1425-1442, 2020.

[35] G. Rasool, A. Shafiq, and D. Baleanu, "Consequences of soretdufour effects, thermal radiation, and binary chemical reaction on Darcy forchheimer flow of nanofluids," Symmetry, vol. 12, no. 9, p. 1421, 2020.

[36] T. Hayat, Z. Bashir, S. Qayyum, and A. Alsaedi, "Nonlinear radiative flow of nanofluid in presence of gyrotactic microorganisms and magnetohydrodynamic," International Journal of Numerical Methods for Heat and Fluid Flow, 2019.

[37] M. Ramzan, H. Gul, and J. D. Chung, "Double stratified radiative Jeffery magneto nanofluid flow along an inclined stretched cylinder with chemical reaction and slip condition," The European Physical Journal Plus, vol. 132, no. 11, pp. 1-17, 2018.

[38] T. Hayat, M. I. Khan, M. Waqas, and A. Alsaedi, "Newtonian heating effect in nanofluid flow by a permeable cylinder," Results in Physics, vol. 7, pp. 256-262, 2018.

[39] M. Suleman, M. Ramzan, S. Ahmad, and D. Lu, "Numerical simulation for homogeneous-heterogeneous reactions and Newtonian heating in the silver-water nanofluid flow past a nonlinear stretched cylinder," Physica Scripta, vol. 94, no. 8, 2019. 